\title{
Disk dwarf galaxy as the progenitor of the Andromeda giant stream
}

\author{
Takanobu Kirihara ${ }^{1}$, Yohei Miki ${ }^{2}$, Masao Mori ${ }^{1,2}$ \\ and Toshihiro Kawaguchi ${ }^{3}$ \\ ${ }^{1}$ Faculty of Pure and Applied Physics, University of Tsukuba, Tennodai 1-1-1, Tsukuba, \\ Ibaraki, Japan \\ email: kirihara@ccs.tsukuba.ac.jp \\ ${ }^{2}$ Center for Computational Sciences, University of Tsukuba, Tennodai 1-1-1, Tsukuba, Ibaraki, \\ Japan \\ ${ }^{3}$ Sapporo Medical University, S1W17, Chuoh-ku, Sapporo, Hokkaido, Japan
}

\begin{abstract}
We present a study of the morphology of a progenitor galaxy that has been disrupted and formed a giant southern stellar stream in the halo of Andromeda galaxy(M31). N-body simulations of a minor merger of M31 with a dwarf galaxy suggest that the progenitor's rotation plays an important role in the formation of an asymmetric surface brightness distribution of the stream.
\end{abstract}

Keywords. galaxies: individual (M31), galaxies: interactions, galaxies: kinematics and dynamics

\section{Introduction}

As represented by a giant southern stream (GSS), recent deep and wide observations with optical wavelength have revealed a wealth of substructures in the halo of M31 (Ibata et al. 2001; Martin et al. 2013). Observed properties of the GSS and north-eastern and western stellar shells have been reproduced in detail with $N$-body simulations of a minor merger that has occurred about 700 Myr ago (Fardal et al. 2007; Mori \& Rich 2008; Miki et al. 2014; Kirihara et al. 2014). The total stellar mass of the progenitor has been estimated $M_{*}>10^{8} \mathrm{M}_{\odot}$ (Font et al. 2006).

We focus on an asymmetric surface brightness distribution of the GSS that has been firstly suggested by McConnachie et al. 2003. We analyzed RGB star count map (Irwin et al. 2005) and obtained azimuthal surface brightness profile of the GSS (Fig. 1). The stellar density of the GSS decreases sharply at the eastern side of the GSS from the most luminous direction. On the other hand, the western side of the GSS extends widely. So far, any simulations assuming a merger of a spherical non-rotating progenitor failed to reproduce this characteristic structure of the GSS (e.g., Fig. 1).

\section{Methods and Results}

We examine the collision of the disk satellite galaxy and M31 using $N$-body simulation. The initial stable model of satellite galaxies with a disk, a bulge, and a dark matter halo is generated by GalactICS (Kuijken \& Dubinski 1995). In this paper, we especially focus on the inclination $(\theta, \phi)$ of the spin axis of the progenitor's disk. Following previous studies, a fixed-potential of M31 and an orbit of the progenitor are taken from Fardal et al. 2007. $N$-body simulations are carried out on the T2K-Tsukuba, HA-PACS, and COMA system in Center for Computational Sciences, University of Tsukuba.

We compare the observed asymmetric spatial structures of the GSS with that of the results of our simulations using $\chi^{2}$ analysis. Fig. 2 shows a $\chi^{2}$ map of the comparison 

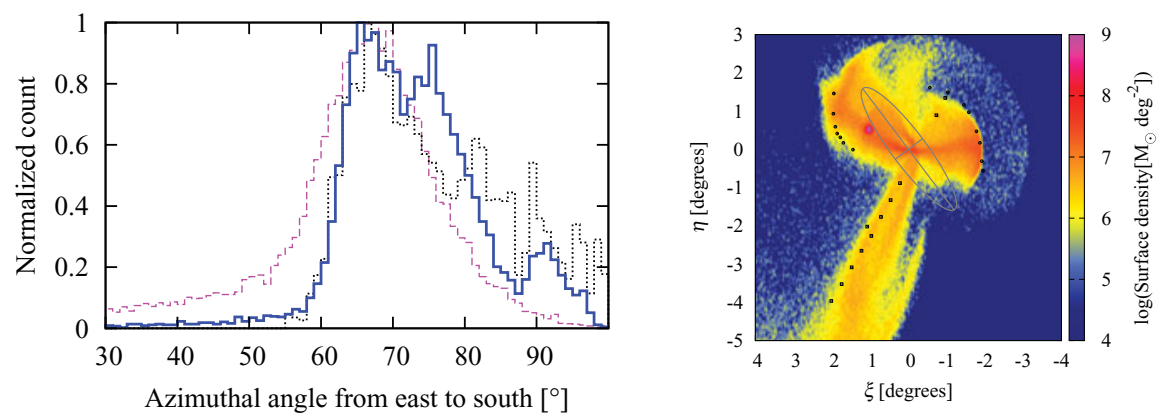

Figure 1. Left: Azimuthal star counts distribution of the GSS. Each line corresponds the observed data (black dotted), best-fitting model (blue solid), and spherical progenitor model (magenta dashed), respectively. Right: Snapshot of the surface density distribution of the disrupted progenitor. The inclined elliptical line describes the shape of the M31 disk. Square symbols and circles are observed fields of the GSS and edges of the shells ( Font et al. 2006; Fardal et al. 2007), respectively.

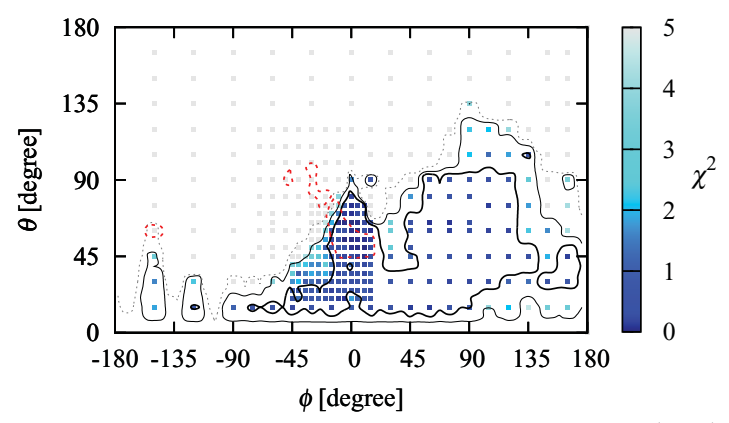

Figure 2. $\chi^{2}$ map for the eastern width of the GSS on the plane $(\theta, \phi)$. The squares indicate the simulated parameters. Black lines describe 1,2 , and $3 \sigma$ confidence intervals, and the dotted red line is $1 \sigma$ level, in which the brightest angle of the GSS is reproduced.

for the narrow eastern width from the brightest direction of the GSS. The most region that the $\chi^{2}$ value is smaller than $1.0(\nu=2 ; 1 \sigma$ confidence interval) corresponds to the disk with anti-clockwise rotation. Fig. 1 presents one of the best-fitting models; the left panel shows an azimuthal density profile of the GSS, and the right panel describes a snapshot of surface density distribution. It is clear that our simulation nicely reproduced the observed asymmetric structure of the GSS.

We conclude that the rotation of the progenitor was crucial for the formation of the asymmetric structure of the GSS, and most likely the progenitor of the GSS was a disk dwarf galaxy.

\section{References}

Fardal, M. A., Guhathakurta, P., Babul, A., \& McConnachie, A. W. 2007, MNRAS, 380, 15

Font, A. S., Johnston, K. V., Guhathakurta, P., Majewski, S. R., \& Rich, M. 2006, AJ, 131, 1436

Ibata, R., Irwin, M., Lewis, G., Ferguson, A., \& Tanvir, N. 2001, Nature, 412, 49

Kirihara, T., Miki, Y., \& Mori, M. 2014, PASJ, 66, L10

Kuijken, K. \& Dubinski, J. 1995, MNRAS, 277, 1341

Martin, N. F. et al., 2013, ApJ, 776, 80

McConnachie, A. W., Irwin, M., Ibata, R., Ferguson, A., Lewis, G., \& Tanvir, N., 2003, MNRAS, 343,1335

Miki, Y., Mori, M., Kawaguchi, T., \& Saito, Y. 2014, ApJ, 783, 87

Mori, M. \& Rich, M. 2008, ApJ, 674, 77 